\title{
VALORACIÓN PSICOMÉTRICA DE LOS COMPONENTES FÍSICOS (CSF) Y MENTALES (CSM) DEL SF-36 EN PACIENTES INSUFICIENTES RENALES CRÓNICOS EN TRATAMIENTO CON HEMODIÁLISIS
}

\author{
PSYCHOMETRIC ASSESMENT OF THE PHYSICAL AND MENTAL \\ COMPONENTS OF THE SF-36 IN CHRONIC RENAL INSUFFICIENCY \\ FAILURE PATIENTS IN TREATMENT WITH HAEMODYALISIS
}

\author{
Magali Rodríguez VidaL ${ }^{*}$ \\ Manuel Merino Escobar ${ }^{* *}$ \\ Manuel Castro Salas ${ }^{* *}$
}

\section{RESUMEN}

La calidad de vida relacionada con la salud es un constructo que se está utilizando cada vez con más frecuencia para evaluar las variables relacionadas con la salud desde la perspectiva del individuo. En este trabajo se analizaron las características descriptivas de las escalas resumidas de componentes de salud físico (CSF) y componentes de salud mental (CSM), provenientes del SF-36 V2 en pacientes insuficientes renales crónicos terminales sometidos a tratamiento con hemodiálisis. Se trata de un estudio cuantitativo de corte transversal cuya población estuvo compuesta por los pacientes de la patología antes citada controlados en los centros de diálisis de la Comuna de Concepción, Chile, en el año 2005. La muestra incluyó a 270 pacientes, que corresponden a un $65 \%$ del universo. El instrumento principal utilizado estuvo constituido por el SF-36, para medir la variable principal Calidad de Vida y se agregaron variables sociodemográficas. El instrumento demostró una alta confiabilidad para los componentes de salud física y mental con un alfa de Cronbach de 0,86 para cada uno. En términos generales los instrumentos CSF y CSM provenientes del SF-36 permiten capturar apropiadamente la situación de vida por la que están atravesando las personas, como es el caso de los pacientes nefrópatas en tratamiento con hemodiálisis, que fueron objeto de este estudio.

Palabras claves: Calidad de vida, hemodiálisis, psicometría, SF-36 V2.

\begin{abstract}
The quality of life related to health is a construct that is being used with increasing frequency to evaluate the variables related to health from the perspective of the individual. In this study we analyzed the characteristics of the summarized descriptive scales of physical health components (CSF) and mental health components (MSC) from the SF-36 V2 in terminal patients with chronic renal insufficiency treated with haemodialysis. This is a cross sectional quantitative study with a population of patients with the previously quoted pathology controlled in the dialysis facilities in the area of Concepcion, Chile in 2005. The sample consisted of 270 patients, which is $65 \%$ of the universe. The main instrument was the SF-36 for measuring the quality of life main variable. Socio-demographic variables were added. The instrument showed a high reliability for the components of physical and mental health with a Cronbach's alpha of 0.86 , for each one. In general terms, the instruments CSF and MSC from the SF-36 properly capture the life conditions that people are experiencing, as is the case with nephropath patients undergoing haemodialysis treatment, who were the subject of this study.
\end{abstract}

Keywords: Quality of life, haemodialysis, psychometry, SF-36-V2

Fecha recepción: 25/03/08 Fecha aceptación: 12/03/09

\footnotetext{
* Profesor asistente Magíster en Enfermería, especialista en Diálisis y Trasplante Renal, Universidad de Concepción, Dirección: Roosevelt s/n Concepción-Chile, email: magrodri@udec.cl

${ }^{* *}$ Profesor titular, sociólogo, Magíster en Ciencias Sociales, PhD in Sociology, Facultad de Ciencias Sociales, Departamento de Sociología, Universidad de Concepción, Chile, jmerino@udec.cl

**** Profesor asociado, Magíster en Enfermería, Sociólogo, Universidad de Concepción, email: mcastro@udec.cl
} 


\section{INTRODUCCION}

El interés en evaluar la calidad de vida relacionada con la salud, en pacientes con enfermedad renal terminal, se inició en la década de los ochenta(1). Estos estudios se focalizaron en la descripción de la calidad de vida de pacientes tratados con diferentes terapias de reemplazo renal, hemodiálisis, diálisis peritoneal y trasplante(2). La calidad de vida relacionada con salud se ha usado para valorar los efectos de la enfermedad y los resultados de tratamientos. Permite además conocer la valoración subjetiva y multidimensional del estado de salud de las personas(3). En la actualidad la salud percibida es uno de los indicadores de salud más utilizados en el tratamiento integral de la enfermedad(4).

La utilización de instrumentos que incorporan la percepción del estado de salud de los individuos en su vida diaria, está siendo objeto de un interés cada vez mayor en la investigación en salud(5). Actualmente existe una amplia gama de instrumentos para valorar la calidad de vida. Su uso intensivo en diferentes contextos, circunstancias y procedimientos tradicionales aseguran la calidad de sus características psicométricas(6).

El SF-36 es una medida multipropósito de calidad de vida que ha sido usada para comparar poblaciones generales y específicas, el impacto de la enfermedad y los beneficios para la salud de una amplia gama de tratamientos $(7,17)$.

Este instrumento últimamente ha permitido el cálculo de puntuaciones de resumen como son el componente físico (PCS) y el componente mental (MCS), que son calculados a partir de las ocho escalas usando algoritmos recomendados por los autores(8).

La patología renal terminal constituye un gran problema de salud pública, por las elevadas tasas de morbilidad y mortalidad que origina, agregándose un impacto negativo sobre la calidad de vida de sus portadores. Quienes cursan esta enfermedad presentan las manifes- taciones características del síndrome urémico, por lo que necesitan terapia de reemplazo para mantenerse con vida. La hemodiálisis se usa con el fin de prolongar la vida, no obstante va acompañada de un deterioro de la calidad de vida de estos pacientes(9).

El propósito de este estudio es analizar psicométricamente el comportamiento de las medidas de resumen físicas (CSF) y mentales (CSM) del SF- 36 cuando son aplicados a pacientes con insuficiencia renal crónica terminal, sometidos a tratamiento con hemodiálisis.

\section{MATERIAL Y METODO}

La presente investigación es un estudio cuantitativo de corte transversal(16). La población estuvo constituida por los pacientes con insuficiencia renal crónica en tratamiento con hemodiálisis, de la comuna de Concepción el año 2005. La muestra de tipo probabilística estuvo conformada por 270 pacientes que corresponden a un $65,8 \%$ del universo. Las unidades de análisis fueron pacientes de los centros de diálisis que autorizaron entrevistar a sus usuarios y que daban garantías de tratamiento adecuado, contando con tecnología de última generación. Previa lectura y firma del consentimiento informado por parte de los pacientes usuarios se procedió a aplicar el cuestionario.

Los criterios de exclusión utilizados consideraron: menos de tres meses de tratamiento sustitutivo, condiciones psíquicas inadecuadas (medido a través del minimental test) y ceguera.

El instrumento final estuvo constituido por el SF-36, que mide la variable principal del estudio, calidad de vida y además se incorporaron algunas variables sociodemográficas (sexo, edad, estado civil, escolaridad, actividad laboral, religión y previsión).

Para su administración se contó con la participación de un encuestador previamente adiestrado (interno de enfermería), quien 
aplicó el instrumento durante la sesión de diálisis.

Para el procesamiento y análisis estadístico de los datos se utilizó el Statistical Analysis System (SAS) en versión 9.2 para Windows XP.

Los estadígrafos utilizados para la valoración de la escala fueron Media, Desviación Estándar, Alpha de Cronbach y T-Student.

El cuestionario genérico de Calidad de Vida (SF-36 ) ha sido ampliamente utilizado a nivel internacional y fue diseñado para evaluar diferentes aspectos relacionados con la salud, desde la perspectiva del individuo. Originalmente fue desarrollado por Ware y Gandek(10) y posteriormente adaptado y validado en España por J. Alonso y col.

Con este constructo se han medido conceptos genéricos de salud relevantes para el individuo, en distintos grupos etáreos, estados de enfermedad y grupos de tratamiento. Proporciona un método seguro, eficiente y sicométricamente sólido para medir la salud desde el punto de vista del paciente, puntuando respuestas estandarizadas a preguntas estandarizadas(11). El cuestionario abreviado SF-36 se estandarizó en 1996 (12). El procedimiento permite medir la variable Calidad de Vida en base a una escala estructurada en ocho dimensiones, con distintos formatos de respuesta, puntuadas en un formato Likert. Las dimensiones son las siguientes: Función Física, Rol Físico, Dolor Corporal, Salud General, Vitalidad, Función Social, Rol Emocional y Salud Mental. El sistema estandarizado de puntuación da por resultado un perfil de ocho escalas de puntuación. La estandarización del contenido y puntuación son esenciales para la interpretación del SF-36 y hace posible comparaciones significativas de los resultados. Las reglas de puntuación son apropiadas para todos los ítemse y las opciones de respuesta del SF-36(13).

Para cada dimensión los ítemes se codifican, agregan y transforman en una escala que tiene un rango de 0 a 100, donde 0 es el peor resultado y 100 el mejor. Sus ítemes detectan tanto estados positivos como negativos de la salud. Este cuestionario se desarrolló a partir de una extensa batería, que incluía cuarenta conceptos relacionados con salud(15) Para crear el cuestionario de una manera eficiente, se seleccionó el mínimo número de conceptos necesarios para mantener la validez y las características operativas del cuestionario inicial. Como se indica anteriormente, el cuestionario final cubre 8 dimensiones del estado de salud y su contenido se centra en el estado funcional y el bienestar emocional(14).

Una descripción sintética de las dimensiones que incluye el SF-36 es:

Función física: Es el grado en que la salud limita las actividades físicas tales como el autocuidado, caminar, inclinarse, realizar esfuerzos, etc. Consta de 10 ítemes con tres alternativas de respuesta, $($ mucho $=1 ;$ poco $=2 ;$ nada $=3)$, con un rango de 10 a 30 puntos

Rol físico: Es el grado en que la salud física interfiere en el trabajo y en otras actividades diarias, generando un rendimiento menor que el deseado. Tiene cuatro ítemes con dos alternativas de respuesta ( $s i=1 ; n o=2)$, en un rango de 4 a 8 puntos.

Dolor corporal: Es el grado de intensidad del dolor y su efecto. Tiene 2 ítemes con 5 alternativas de respuesta, $($ mucho $=1$; bastante $=2$; regular $=3$; un poco $=4 ;$ nada $=5$ ), con un rango de 2 a 10 puntos.

Salud general: Es una valoración personal de la salud que incluye la salud actual, las perspectivas de salud en el futuro y la resistencia a enfermar. Tiene 5 ítemes y con 5 alternativas de respuesta, $($ todo verdadero $=1$; bastante cierto $=$ 2 ; no lo sé $=3$; bastante falsa $=4$; totalmente falso $=5$ ), en un rango de 5 a 25 puntos.

Vitalidad: Sentimiento de energía y vitalidad frente al sentimiento de cansancio y agotamiento, tiene 4 itemes con 5 alternativas de respuesta $($ en extremo $=5 ;$ mucho $=4$, normal $=3$; $\operatorname{algo}=2$; nada $=1$ ), en un rango de 4 a 20 puntos.

Función Social: Grado en que los problemas de salud interfieren en la vida social. Tiene 2 itemes con 5 alternativas de respuesta (siem- 
pre $=1$; casi siempre $=2$; algunas veces $=3$; sólo algunas vez $=4$; nunca $=5$ ), en un rango de 2 a 10 puntos.

Rol Emocional: Grado en que los problemas emocionales interfieren en el trabajo u otras actividades diarias, incluyendo reducción en el tiempo dedicado a éstas, rendimiento menor y disminución de la intensidad en el trabajo. Tiene 3 ítemes con 2 alternativas de respuesta, $(\mathrm{si}=1$; no $=2$ ), en un rango de 3 a 6 puntos.

Salud Mental: Incluye depresión, ansiedad, control de la conducta y el bienestar. Tiene 5 ítemes con cinco alternativas de respuesta, (siempre $=1$; casi siempre $=2$; algunas veces $=3$; sólo algunas $\mathrm{vez}=4$; nunca $=5$ ), en un rango de 5 a 25 puntos.
La adición de los puntajes entregados por los sujetos a cada uno de los ítemes determina el valor que se obtiene en cada dimensión. La calidad de vida operacionalmente hablando es la sumatoria global de los puntajes que los sujetos otorgan a cada uno de los 36 ítemes que componen la escala.

Las subescalas del SF-36 constituidas por las ocho dimensiones descritas están ordenadas en su mayoría de manera que a mayor puntuación mejor es el estado de salud, exceptuando las dimensiones dolor corporal, salud general y vitalidad. La Tabla 1 contiene el número de ítems incluidos en las diferentes sub-escalas del cuestionario, así como una breve descripción del significado de sus puntuaciones altas y bajas.

Cuadro 1: Itemes y significado de las puntuaciones altas y bajas del SF- 36.

\begin{tabular}{|c|c|c|c|}
\hline Dimensión & $\mathbf{N}^{\circ}$ ítemes & Peor puntuación 0 & Mejor puntuación 100 \\
\hline $\begin{array}{c}\text { Función } \\
\text { física }\end{array}$ & 10 & $\begin{array}{c}\text { Muy limitado para llevar a cabo todas } \\
\text { las actividades físicas incluido bañarse } \\
\text { o ducharse debido a la salud }\end{array}$ & $\begin{array}{c}\text { Lleva a cabo todo tipo de activi- } \\
\text { dades fisicas, incluidas las más } \\
\text { vigorosas sin ninguna limitación } \\
\text { debido a su salud }\end{array}$ \\
\hline Rol físico & 4 & $\begin{array}{c}\text { Problemas con el trabajo u otras acti- } \\
\text { vidades diarias debido a salud física }\end{array}$ & $\begin{array}{c}\text { Ningún problema con el traba- } \\
\text { jo u otras actividades diarias } \\
\text { debido a la salud física }\end{array}$ \\
\hline Dolor corporal & 2 & $\begin{array}{c}\text { Dolor muy intenso y extremadamente } \\
\text { limitante }\end{array}$ & $\begin{array}{c}\text { Ningún dolor ni limitaciones } \\
\text { debidas a él }\end{array}$ \\
\hline Salud general & 5 & $\begin{array}{c}\text { Evalúa como mala la propia salud y } \\
\text { cree posible que empeore }\end{array}$ & $\begin{array}{c}\text { Evalúa la propia salud como } \\
\text { excelente }\end{array}$ \\
\hline Función social & 2 & $\begin{array}{c}\text { Interferencia extrema y muy frecuente } \\
\text { con las actividades sociales normales } \\
\text { debido a problemas físicos o emocio- } \\
\text { nales }\end{array}$ & $\begin{array}{c}\text { Se siente cansado y exhausto todo el } \\
\text { sociales normales sin ninguna } \\
\text { interferencia debido a problemas } \\
\text { físicos o emocionales }\end{array}$ \\
\hline Rol emocional & 3 & $\begin{array}{c}\text { Problemas con el trabajo y otras } \\
\text { actividades diarias debido a problemas } \\
\text { emocionales }\end{array}$ & $\begin{array}{c}\text { Ningún problema con el trabajo } \\
\text { y otras actividades diarias debi- } \\
\text { do a problemas emocionales }\end{array}$ \\
\hline Salud mental & 5 & $\begin{array}{c}\text { Sentimiento de angustia y depresión } \\
\text { durante todo el tiempo }\end{array}$ & $\begin{array}{c}\text { Sentimiento de felicidad, tran- } \\
\text { quilidad y calma durante todo el } \\
\text { tiempo }\end{array}$ \\
\hline Item de transición \\
de salud
\end{tabular}


Mediante la combinación de las puntuaciones de cada dimensión el cuestionario permite el cálculo de 2 componentes sintéticos: el físico (CSF) y el mental (MCS).

Análisis de tipo factoriales efectuados en diversas poblaciones han detectado elevada confiabilidad de las 8 escalas, del orden de un 80 a $85 \%$, lo que ha permitido la construcción de medidas de resumen. Estas medidas hacen posible reducir el número de comparaciones estadísticas implicadas en el análisis del SF-36 de ocho dimensiones a dos, sin pérdida de su potencial para distinguir entre resultados de salud física y mental, sea en estudios transversales o longitudinales (12).

Los análisis factoriales realizados en muestras americanas y otras muestras representativas de la población general de 9 países europeos del proyecto IQOLA demostraron que las 8 escalas definen adecuadamente los 2 componentes principales de salud: el CSF y CSM. Los pasos principales de los algoritmos de cálculo de las puntuaciones de las 2 medidas son:

a) estandarización de las 8 escalas del SF-36, con la media y desviación estándar de la población general.

b) ponderación de las escalas aplicando los pesos factoriales obtenidos en el análisis de componentes principales.

c) agregación de las escalas y transformación, para obtener una media de 50 y una desviación estándar de 10 en la población general.

\section{RESULTADOS}

La Tabla 2 exhibe los promedios y dispersiones de cada ítem componente de la escala SF-36 ordenados por subdimensión en su aplicación directa. La variable dependiente Calidad de Vida en la subescala función física (ff), los cuatro ítemes que miden actividad física intensa tienen valores promedios inferiores a dos puntos, lo que significa que estos pacientes tienen severas limitaciones para el desarrollo de sus actividades físicas cotidianas. Los ítemes que miden actividad física moderada concentran valores promedio ligeramente superiores a dos puntos, lo que expresa un nivel de limitación física de nivel intermedio. En general, puede inferirse de los datos que los pacientes renales tienen una función física bastante precaria o muy limitada.

Tabla 2: Estadística descriptiva de las preguntas del Cuestionario SF-36

\begin{tabular}{|l|c|c|}
\hline Itemes & Media & DESV st \\
\hline FFAct. Vigorosa & 1.24 & 0.51 \\
\hline Act. Moderada & 2.03 & 0.85 \\
\hline Levantar bolsa compras & 2.06 & 0.85 \\
\hline Subir varios Pisos & 1.66 & 0.81 \\
\hline Subir un piso & 2.24 & 0.82 \\
\hline Agacharse, arrodillarse & 1.96 & 0.85 \\
\hline Caminar 1 Kilómetro & 1.93 & 0.90 \\
\hline Caminar algunas Cuadras & 2.28 & 0.84 \\
\hline Caminar 1 cuadra & 2.06 & 0.92 \\
\hline Bañarse, Vestirse & 2.68 & 0.66 \\
\hline Rf. Disminuyó el tiempo & 1.52 & 0.50 \\
\hline Menos Cumplimiento & 1.35 & 0.47 \\
\hline Algo Limitado & 1.44 & 0.49 \\
\hline Tuvo Dificultad & 1.42 & 0.49 \\
\hline DC Magnitud del dolor & 3.27 & 1.33 \\
\hline
\end{tabular}




\begin{tabular}{|l|l|l|}
\hline Interferencia del dolor & 3.76 & 1.42 \\
\hline SG un año atrás & 3.11 & 1.24 \\
\hline Se enferma fácil & 2.36 & 1.21 \\
\hline Tan saludable como otros & 2.64 & 1.07 \\
\hline Salud va a empeorar & 2.27 & 1.23 \\
\hline Salud excelente & 2.18 & 0.81 \\
\hline Vit. Animado & 3.26 & 1.25 \\
\hline Con energía & 3.07 & 1.30 \\
\hline Agotado & 2.99 & 1.19 \\
\hline Cansado & 2.80 & 1.15 \\
\hline FS. Alcance social & 3.21 & 1.57 \\
\hline Magnitud tiempo social & 3.73 & 1.44 \\
\hline RE.Disminuyó el tiempo & 1.62 & 0,48 \\
\hline Menos cumplimiento & 1.57 & 0.49 \\
\hline No cuidadoso & 1.59 & 0.49 \\
\hline SM. nervioso & 3.21 & 1.37 \\
\hline Desanimado & 3.65 & 1.36 \\
\hline Tranquilo & 3.78 & 1.11 \\
\hline Triste & 3.19 & 1.34 \\
\hline Feliz & 3.44 & 1.30 \\
\hline
\end{tabular}

FUENTE: Calidad de Vida en Pacientes Portadores de Insuficiencia Renal Crónica Terminal y en Tratamiento con Hemodiálisis. Proyecto Diuc. Magali Rodríguez. 2005.

La subescala rol físico mide el impacto de las limitaciones físicas sobre las actividades cotidianas, en términos de la conciencia del paciente renal del grado en que sus limitaciones físicas comienzan a restringir sus roles habituales. La mayoría reconoce que su enfermedad comienza seriamente a restringir su tiempo de trabajo útil o a afectar el cumplimiento de sus tareas productivas, debido a las limitaciones y dificultades que impone la enfermedad.

La subescala dolor corporal permite concluir que, consistente con las limitaciones físicas anteriores, los niveles de dolor reconocidos por los pacientes renales son moderados a serios.

Sin embargo, la dimensión salud general, que permite conocer la valoración de su salud hecha por el propio paciente, revela que éste no se valora en buenos niveles actuales y es bastante pesimista respecto del futuro, puesto que una gran mayoría perciben que la salud va a empeorar.

La medición de la vitalidad informada es bastante expresiva, aunque los pacientes tiendan a definirse como animados y con energía se reconocen físicamente agotados y con niveles elevados de cansancio.

En cuanto al grado en que la enfermedad interfiere en la vida social del paciente, la mayoría reconoce un impacto social moderado proveniente de la condición de enfermo.

El nivel en que la enfermedad interfiere con las emociones del paciente parece ser menor que lo esperado, puesto que sus respuestas puntúan más cerca de la inexistencia de problemas en esta dimensión, que en el reconocimiento de interferencias de origen emocional.

La subescala salud mental $(\mathrm{sm})$ incluye el reconocimiento del efecto que puede tener la enfermedad sobre la ansiedad, depresión y ánimo del paciente. En consistencia con la subescala rol emocional (re) los pacientes no consideran que la enfermedad esté interfiriendo con sus niveles de ansiedad, estados de humor, ánimo o felicidad. 
En resumen el examen de los ítemes que les de dificultad, en las dimensiones psicológicas componen la escala SF-36 de calidad de vida per- y sociales estas personas no puntúan diferente mite concluir que, aunque en las dimensiones de lo que obtendría una persona normal que no físicas los pacientes reconocen los más altos nive- sufre de insuficiencia renal crónica terminal.

Tabla 3: Correlaciones de las preguntas de la escala SF-36

\begin{tabular}{|c|c|c|c|c|c|c|c|c|}
\hline Itemes & FF & RF & $\mathrm{DC}$ & SG & VT & FS & RE & SM \\
\hline FFAct. Vigorosa & 0.54 & & & & & & & \\
\hline Act. Moderada & 0.81 & & & & & & & \\
\hline Levantar bolsa compras & 0.75 & & & & & & & \\
\hline Subir varios Pisos & 0.76 & & & & & & & \\
\hline Subuir un piso & 0.84 & & & & & & & \\
\hline Agacharse, arrodillarse & 0.71 & & & & & & & \\
\hline Caminar 1 Kilómetro & 0.88 & & & & & & & \\
\hline Caminar algunas Cuadras & 0.87 & & & & & & & \\
\hline Caminar 1 cuadra & 0.87 & & & & & & & \\
\hline Bañarse, Vestirse & 0.58 & & & & & & & \\
\hline Rf. Disminuyó el tiempo & & 0.76 & & & & & & \\
\hline Menos Cumplimiento & & 0.80 & & & & & & \\
\hline Algo Limitado & & 0.82 & & & & & & \\
\hline Tuvo Dificultad & & 0.84 & & & & & & \\
\hline DC Magnitud del dolor & & & 0.90 & & & & & \\
\hline Interferencia del dolor & & & 0.91 & & & & & \\
\hline SG un año atrás & & & & 0.64 & & & & \\
\hline Se enferma fácil & & & & 0.72 & & & & \\
\hline Tan saludable como otros & & & & 0.61 & & & & \\
\hline Salud va a empeorar & & & & 0.76 & & & & \\
\hline Salud excelente & & & & 0.61 & & & & \\
\hline Vit Animado & & & & & 0.74 & & & \\
\hline Con energía & & & & & 0.76 & & & \\
\hline Agotado & & & & & 0.72 & & & \\
\hline Cansado & & & & & 0.79 & & & \\
\hline FS Alcance social & & & & & & 0.83 & & \\
\hline Magnitud tiempo social & & & & & & 0.80 & & \\
\hline RE Disminuyó el tiempo & & & & & & & 0.90 & \\
\hline Menos cumplimiento & & & & & & & 0.89 & \\
\hline No cuidadoso & & & & & & & 0.92 & \\
\hline SM nervioso & & & & & & & & 0.73 \\
\hline Desanimado & & & & & & & & 0.80 \\
\hline Tranquilo & & & & & & & & 0.69 \\
\hline Triste & & & & & & & & 0.80 \\
\hline Feliz & & & & & & & & 0.71 \\
\hline
\end{tabular}

Fuente: Idem Tabla 1 
Tabla 4: Alpha de Cronbach por subescalas del SF-36.

\begin{tabular}{|l|c|c|}
\hline Escalas & Items & Alpha de Cronbach \\
\hline Función Física & 10 & 0.92 \\
\hline Rol Físico & 4 & 0.82 \\
\hline Dolor Corporal & 2 & 0.80 \\
\hline Salud General & 5 & 0.69 \\
\hline Vitalidad & 4 & 0.75 \\
\hline Función Social & 2 & 0.50 \\
\hline Rol Emocional & 3 & 0.89 \\
\hline Salud Mental & 5 & 0.80 \\
\hline
\end{tabular}

Fuente: idem tabla 1

Las tablas tres y cuatro deben ser examinadas simultáneamente porque, mientras una describe la correlación entre cada ítem y el puntaje total de la subescala, la segunda entrega para cada dimensión un índice resumido de su nivel interno de consistencia.

La escala de funcionamiento físico (ff) tiene una elevada confiabilidad (alpha de Cronbach de 0.92) y la correlación de los ítemes con la subescala es también muy convergente, con la excepción de los ítemes referentes a la ejecución de actividad física vigorosa, que siendo ésta mayoritariamente reconocida como un problema por los pacientes, no comparte el sentido positivo que los pacientes atribuyen a los otros componentes de la dimensión ff. En cambio el ítem que establece bañarse o vestirse por sí mismo como un problema de funcionamiento físico para estas personas, fue casi unánimemente reconocido como no siendo un problema, puesto que los pacientes reconocen un alto grado de independencia funcional. En este sentido la casi unanimidad de los ítemes también perturba la correlación del ítem con el puntaje total de la dimensión. El examen del resto de las correlaciones entre los ítemes y su puntaje total, en la dimensión, revela marcada consistencia, por tanto excelentes niveles de confiabilidad.

Sólo dos comentarios deberían ser hechos en este contexto. La escala con menos alpha de Cronbach, menos confiabilidad interna, resultó ser la subescala de función social (fs). Sin embargo esta escala sólo tiene dos ítemes, lo que impide examinar adecuadamente su tendencia.

La escala de salud general (sg), que valora por parte del paciente su condición pasada, actual y próxima de salud tiene un nivel relativamente moderado de correlación ítemescala (sobre 0,60), estos niveles relativamente moderados de correlación entre los ítemes y el total se expresan fundamentalmente porque la imagen de salud de los pacientes captura sólo un nivel intermedio de optimismo. Eso es lo que se expresa en la confiabilidad de 0,69 para la dimensión.

Tabla 5: Estadísticas descriptivas de las escalas del SF-36(6).

\begin{tabular}{|l|c|c|c|c|c|c|c|c|}
\hline & FF & RF & DC & SG & VT & FS & RE & SM \\
\hline $\mathrm{N}^{\circ}$ ítemes & 10 & 4 & 2 & 5 & 4 & 2 & 3 & 5 \\
\hline Media & 50.8 & 43.5 & 50.3 & 37.9 & 40.7 & 61.7 & 59.6 & 49.1 \\
\hline IC (95\%) & 95 & 100 & 80 & 75 & 75 & 100 & 100 & 80 \\
\hline Mediana & 55.0 & 25.0 & 50 & 35 & 40 & 62.5 & 100 & 48 \\
\hline
\end{tabular}

Fuente: idem tabla 1 
En la Tabla 5 se puede observar el comportamiento de los ítemes en las subescalas con puntajes aditivos estandarizados, esto significa que los puntajes varían entre 0 y 100 en función de extremos que son de peor a mejor bienestar general, como producto de la enfermedad. Los puntajes estandarizados permiten observar que las dimensiones más deprimidas en estos pacientes son la salud general (37.9), vitalidad (40.7) y el rol físico. Estas tres dimensiones indican que valoran su salud como deteriorada significativamente.

Los puntajes en las otras dimensiones de la escala van desde la zona intermedia a la moderadamente positiva. En las subescalas de funcio- namiento físico, dolor corporal, salud mental, estas personas están en el medio de la escala que va desde la buena a la mala calidad de vida; en sólo dos dimensiones, respuesta emocional y funcionamiento social, los pacientes renales puntúan ligeramente positivo en la escala.

En términos generales, la conclusión es que la escala SF-36, con sus subescalas, captura muy adecuadamente la mala condición general de la salud de este grupo de personas crónicamente enfermas.

En la Tabla 6 se presentan los resultados de las escalas resumidas de calidad de vida (SF-36) en sus dos componentes, físico (CSF) y mental (CSM).

Tabla 6: Resumen de las dimensiones del SF-36 V2, presentando el CSF y el CSM.

\begin{tabular}{|c|c|c|c|c|c|c|c|c|c|}
\hline \multirow[b]{2}{*}{ Escalas } & \multicolumn{2}{|c|}{ Correlación } & \multicolumn{2}{|c|}{$\begin{array}{l}\text { Número } \\
\text { de }\end{array}$} & \multirow[b]{2}{*}{ Mean } & \multirow[b]{2}{*}{ SD } & \multirow{2}{*}{$\begin{array}{l}\text { Con- } \\
\text { fiabili- } \\
\text { dad }\end{array}$} & \multicolumn{2}{|c|}{ Definición(\% observada) } \\
\hline & CSF & CSM & Item & Niveles & & & & Puntaje más bajo & Puntaje más alto \\
\hline $\begin{array}{l}\text { Función } \\
\text { física }\end{array}$ & .87 & .57 & 10 & 21 & 50.8 & 31.3 & 0.92 & $\begin{array}{l}\text { Muy limitado en la } \\
\text { realización de } \\
\text { todas las } \\
\text { actividades físicas }\end{array}$ & $\begin{array}{l}\text { Realización de todo tipo } \\
\text { de actividades físicas } \\
\text { incluyendo las más } \\
\text { vigorosas, sin limitaciones } \\
\text { debidas a la salud }\end{array}$ \\
\hline Rol físico & .51 & .42 & 4 & 5 & 43.5 & 40.0 & 0.83 & $\begin{array}{l}\text { Problemas con el } \\
\text { trabajo u otras } \\
\text { actividades diarias } \\
\text { como resultado de } \\
\text { la salud física }\end{array}$ & $\begin{array}{l}\text { Sin problemas con el } \\
\text { trabajo u otras } \\
\text { actividades diarias }\end{array}$ \\
\hline $\begin{array}{l}\text { Dolor } \\
\text { Corporal }\end{array}$ & .52 & .26 & 2 & 11 & 50.32 & $\begin{array}{c}25.1 \\
7\end{array}$ & 0.80 & $\begin{array}{l}\text { Dolor muy severo y } \\
\text { extremadamente } \\
\text { limitante }\end{array}$ & $\begin{array}{l}\text { Sin dolor o limitaciones } \\
\text { debidas a él }\end{array}$ \\
\hline $\begin{array}{l}\text { Salud } \\
\text { general }\end{array}$ & .67 & .54 & 5 & 21 & 37.9 & 18.9 & 0.69 & $\begin{array}{l}\text { Evalúa la salud } \\
\text { general como } \\
\text { pobre y cree que } \\
\text { podría empeorar }\end{array}$ & $\begin{array}{l}\text { Evalúa la salud personal } \\
\text { como excelente }\end{array}$ \\
\hline Vitalidad & .67 & .81 & 4 & 21 & 40.7 & 18.7 & 0.75 & $\begin{array}{l}\text { Se siente cansado } \\
\text { y desanimado todo } \\
\text { el tiempo }\end{array}$ & $\begin{array}{l}\text { Se siente lleno de vigor y } \\
\text { energía todo el tiempo }\end{array}$ \\
\hline $\begin{array}{l}\text { Función } \\
\text { Social }\end{array}$ & .47 & .72 & 2 & 9 & 61.7 & 30.8 & 100 & $\begin{array}{l}\text { Frecuente } \\
\text { interferencia con } \\
\text { las actividades } \\
\text { sociales normales } \\
\text { debido a problemas } \\
\text { físicos y } \\
\text { emocionales }\end{array}$ & $\begin{array}{l}\text { Realización normal de } \\
\text { actividades sociales sin } \\
\text { interferencias debido a } \\
\text { problemas físicos o } \\
\text { emocionales }\end{array}$ \\
\hline $\begin{array}{l}\text { Rol } \\
\text { Emocional }\end{array}$ & .41 & .65 & 3 & 4 & 59.6 & 44.5 & 0.89 & $\begin{array}{l}\text { Problemas con el } \\
\text { trabajo u otras } \\
\text { actividades diarias } \\
\text { como resultado de } \\
\text { problemas } \\
\text { emocionales }\end{array}$ & $\begin{array}{l}\text { Sin problemas con el } \\
\text { trabajo u otras } \\
\text { actividades diarias }\end{array}$ \\
\hline $\begin{array}{l}\text { Salud } \\
\text { Mental }\end{array}$ & .55 & .89 & 5 & 26 & 49.1 & 19.5 & 0.81 & $\begin{array}{l}\text { Sentimientos de } \\
\text { nerviosismo y } \\
\text { depresión todo el } \\
\text { tiempo }\end{array}$ & $\begin{array}{l}\text { Se siente en paz, feliz y } \\
\text { calmado todo el tiempo }\end{array}$ \\
\hline CSF & & 21 & & & & & & & \\
\hline CSM & & 14 & & & & & & & \\
\hline
\end{tabular}

Note. From Ware, Kosinski, and Keller (1994). 
El componente físico agrupa las cuatro primeras subescalas de funcionamiento físico (ff), rol físico (rf), dolor corporal (dc), y salud general (sg). El componente mental está formado por las subdimensiones de vitalidad (vit), función social (fs), rol emocional ( $\mathrm{rm}$ ) y salud mental (sm). El componente físico (CSF) está compuesto por 21 ítemes, mientras que el componente mental (CSM) por 14. La confiabilidad de las dimensiones resumidas CSF y CSM es muy alta, con un Alpha de Cronbach 0.86.

El análisis de los componentes principales efectuado en nuestros datos ratifica la presencia de estos dos componentes sintéticos, sin embargo la proporción de varianza explicada por ambos componentes es de $55 \%$, lo que indica que la población de pacientes renales estudiada tiene características específicas que hacen que la capacidad explicativa de los componentes no sea en este caso excelente sino sólo regular a bueno.

La intercorrelación de las subescalas con el índice global resulta más satisfactoria; las cuatro subescalas del CSF tienen una correlación con la medida compuesta, mejor que con el CSM. A su vez las cuatro subescalas del CSM correlacionan más alto con su medida agregada, que lo que hacen las subescalas del dominio físico. Por ejemplo, en la Tabla 7 puede observarse que la subescala de función física correlaciona en 0.87 con CSF, su dominio específico, mientras tiene sólo 0.57 con el CSM, el dominio agregado de salud mental.

\section{ANÁLISIS DESCRIPTIVO DE CSF Y CSM}

Es interesante examinar en esta sección el comportamiento descriptivo de los componentes sintéticos de la escala SF-36. La primera escala resumida (CSF) mide el componente asociado a aspectos físicos de la calidad de vida. CSM es la nueva dimensión que concentra exclusivamente aspectos subjetivos o mentales.

En Tabla 7 paneles A y B, se comparan las distribuciones empíricas de los componentes de salud física (CSF) y mental (CSM), por edad y sexo, de los pacientes renales. En la dimensión física (Panel A) en los grupos de edad más jóvenes no existen diferencias de calidad de vida entre hombres y mujeres, en cambio en los grupos de edad superior a 44 años existen claras diferencias de género en cuanto a calidad de vida física. Los hombres declaran mejores puntajes de CSF que las mujeres. En el componente subjetivo mental (CSM) de la calidad de vida se detecta exactamente el mismo patrón que en el componente físico, no existen diferencias significativas entre hombres y mujeres, en calidad de vida, antes de los 44 años. Existen eso sí diferencias estadísticas muy marcadas entre hombres y mujeres en los dos grupos etarios mayores. En los pacientes de mayor edad, los hombres declaran menos problemas asociados a CSM que las mujeres.

El perfil que se obtiene es muy expresivo: en la dimensión física los pacientes de sexo masculino reconocen mejor calidad de vida que las pacientes de sexo femenino, pero esta diferencia es real estadísticamente sólo en los grupos de pacientes de mayor edad. En el componente mental de la calidad de vida la situación es exactamente igual aunque las diferencias entre hombres y mujeres son aún mayores.

En la Tabla 7 se examinan diferencias de calidad de vida física y mental por escolaridad de los pacientes renales, de nuevo controlando el sexo de los enfermos. En el panel de la calidad de vida física (CSF) se observan nítidas diferencias de puntaje entre los pacientes de baja y alta escolaridad. Los pacientes de alta escolaridad tienen mejor valoración de su calidad de vida en sus aspectos físicos, no existiendo diferencias sexuales en este patrón. En el grupo de pacientes de escolaridad baja, simultáneamente, se concentran los puntajes más bajos de percepción de calidad de vida y existen diferencias significativas de esta percepción entre sexos. Las mujeres de menor educación valoran más negativamente su calidad de vida física que los hombres del mismo nivel bajo de educación. 
En el panel B, correspondiente al componente de calidad de vida mental (CSM), se observa el mismo efecto de la escolaridad sobre la calidad de vida mental. El grupo de pacientes de menor educación valora más negativamente su calidad de vida que los pacientes renales más educados, sin embargo en este caso existe una singularidad adicional, en ambos grupos de educación existen diferencias significativas entre hombres y mujeres cuando valoran su calidad de vida, en las dimensiones mentales. Podemos observar que la escolaridad establece, nuevamente, que son los pacientes de sexo femenino quienes valoran más negativamente su calidad de vida mental.

Posteriormente se examinan preliminarmente los efectos de la actividad laboral declarada por los pacientes renales sobre su calidad de vida en ambas dimensiones controlando el sexo de los encuestados. Tanto en CSF como en CSM existe una clara influencia de la actividad laboral sobre la calidad de vida. Los enfermos renales activos laboralmente valoran más positivamente su calidad de vida que los pacientes inactivos. Además se constató profundas diferencias de calidad de vida percibida en ambas dimensiones para ambos sexos. Los pacientes de sexo femenino valoran más negativamente que los de sexo masculino su calidad de vida, tanto en situación de actividad como de inactividad laboral.

Finalmente, los paneles de la Tabla 7 exhiben la comparación efectuada de CSF y CSM en función del estado civil de los pacientes renales, controlando el sexo de los mismos.

En el componente físico (CSF), la principal diferencia observada es entre los pacientes que se declaran sin pareja. En ese grupo existe considerable diferencia de CSF entre hombres y mujeres. Las pacientes de sexo femenino que viven en pareja declaran una calidad de vida física más baja en relación a los hombres con pareja. Las diferencias de puntaje de calidad de vida física entre hombres y mujeres sin pareja son significativas, a un nivel de 2 por 1.000 casos. Eso expresa una considerable diferencia de la calidad de vida entre ambos grupos de género. Esto es aún más sorprendente, puesto que entre los pacientes que viven sin parejas no existen diferencias de calidad de vida entre ambos sexos. Al examinar los puntajes (CSF), de las mujeres que viven en pareja respecto de las que viven sin parejas, existe una considerable diferencia que favorece a los sin pareja. Las mujeres enfermas renales que viven en pareja reconocen a esta condición como uno de los contribuyentes de su mala calidad de vida física.

Tabla 7: CSF y CSM en usuarios portadores de IRCT sometidos a tratamiento con hemodiálisis PANEL A

\begin{tabular}{|c|c|c|c|c|c|c|c|c|}
\hline \multirow[b]{2}{*}{ Edad } & \multicolumn{3}{|c|}{ HOMBRES CSF } & \multicolumn{5}{|c|}{ MUJERES CSF } \\
\hline & $\mathrm{N}$ & MEDIA & DS & $\mathrm{N}$ & MEDIA & DS & T STUDENT & $P$ \\
\hline$<24$ & 5 & 50.6 & 8.3 & 3 & 44.3 & 3.8 & 1.2 & 0.27 \\
\hline$>24-<44$ & 28 & 52.8 & 8.3 & 29 & 49.2 & 9.4 & 1.5 & 0.13 \\
\hline$>44-<64$ & 71 & 46.5 & 8.9 & 57 & 43.1 & 10.5 & 2.0 & 0.04 \\
\hline$>64$ y mas & 37 & 44.8 & 11.5 & 45 & 40.3 & 8.7 & 1.9 & 0.05 \\
\hline \multicolumn{9}{|l|}{ Escolaridad } \\
\hline Baja & 63 & 43.9 & 8.4 & 83 & 41 & 8.9 & 2.0 & 0.04 \\
\hline Alta & 78 & 50.3 & 10.1 & 51 & 47.6 & 10.5 & 1.4 & $0-15$ \\
\hline \multicolumn{9}{|l|}{ S. Laboral } \\
\hline Activo & 62 & 50.3 & 9.4 & 104 & 44.6 & 10.3 & 3.6 & 0.0004 \\
\hline Inactivo & 79 & 45.2 & 9.7 & 30 & 39.7 & $8-0$ & 2.7 & 0.0039 \\
\hline \multicolumn{9}{|l|}{ Estado Civil } \\
\hline Con pareja & 105 & 47.7 & 10.3 & 72 & 41.9 & 9.6 & 3.8 & 0.0002 \\
\hline Sin pareja & 36 & 46.6 & 8.7 & 62 & 45.4 & 10.2 & 0.6 & 0.55 \\
\hline
\end{tabular}


PANEL B

\begin{tabular}{|c|c|c|c|c|c|c|c|c|}
\hline \multirow[b]{2}{*}{ Edad } & \multicolumn{3}{|c|}{ HOMBRES CSM } & \multicolumn{4}{|c|}{ MUJERES CSM } & \multirow[b]{2}{*}{$\mathbf{P}$} \\
\hline & $\mathrm{N}$ & MEDIA & DS & $\mathrm{N}$ & MEDIA & DS & T STUDENT & \\
\hline$<24$ & 5 & 41.4 & 6.3 & 3 & 33.3 & 6.7 & 1.7 & 0.13 \\
\hline$>24-<44$ & 28 & 44.8 & 8.1 & 29 & 44.0 & 6.6 & 0.4 & 0.70 \\
\hline$>44-<64$ & 71 & 42.5 & 9.9 & 57 & 39.0 & 9.9 & 1.9 & 0.05 \\
\hline$>64$ y más & 37 & 43.3 & 11.1 & 45 & 36.2 & 10.9 & 2.8 & 0.005 \\
\hline \multicolumn{9}{|l|}{ Escolaridad } \\
\hline Baja & 63 & 40.0 & 9.5 & 83 & 37.2 & 9.9 & 1.7 & 0.09 \\
\hline Alta & 78 & 45.7 & 9.3 & 51 & 42.1 & 9.3 & 2.1 & 0.03 \\
\hline \multicolumn{9}{|l|}{ S. Laboral } \\
\hline Activo & 62 & 44.8 & 9.9 & 104 & 39.8 & 10.2 & 3.1 & 0.002 \\
\hline Inactivo & 79 & 41.8 & 9.5 & 30 & 36.5 & 8.6 & 2.7 & 0.009 \\
\hline \multicolumn{9}{|c|}{ Estado Civil } \\
\hline Con pareja & 105 & 43.4 & 10.5 & 72 & 37.9 & 10.2 & 3.5 & 0.0006 \\
\hline Sin pareja & 36 & 42.4 & 7.4 & 62 & 40.5 & 9.8 & 0.9 & 0.32 \\
\hline
\end{tabular}

Fuente: idem tabla 1

En el componente mental de calidad de vida, la situación anterior se vuelve a expresar con mayor claridad y peores consecuencias. Entre los pacientes con pareja existe una considerable diferencia de CSM entre hombre y mujeres el sexo femenino reconoce los puntajes más negativos de CSM del estudio y considerablemente inferiores a los de sexo masculino viviendo en parejas. Desde el punto de vista subjetivo de la calidad de vida y la dimensión física no existen diferencias consistentes de calidad de vida total entre hombres y mujeres viviendo solos. La vida en pareja de los pacientes renales perjudica notoriamente al sexo femenino, que empeora su situación de salud al desarrollar percepciones de calidad de vida física y mental muy negativas e inferiores a las de los hombres en la misma condición

\section{CONCLUSIONES Y DISCUSIÓN}

El propósito de este estudio fue analizar el comportamiento descriptivo de las escalas resumidas, CFS y CSM, provenientes del SF-36, en pacientes que sufren insuficiencia renal crónica.

Las medidas sintéticas CSF y CSM, basadas en el SF-36, han sido desarrolladas y aplicadas en los últimos años con el propósito de simplificar y focalizar dimensionalmente las medidas de calidad de vida y así reducir el número de comparaciones estadísticas implicadas en analizar el SF-36 de ocho dimensiones a dos, sin la pérdida de su potencial para distinguir entre resultados de salud física y mental. La idea de los autores consiste en ser capaces de medir la calidad de vida en un componente de salud física y en otro de salud mental que mantenga las propiedades psicométricas del cuestionario original SF-36 ya sea en estudios transversales o longitudinales.

La aplicación en nuestro estudio ratifica el propósito central de Ware y Kosinski: Los componentes capturan adecuadamente la calidad de vida física y mental de los pacientes estudiados.

El componente de salud física (CSF) compuesto por la agregación de las subescalas de funcionamiento físico, rol físico, dolor corporal y salud general, obtiene un promedio de 46.6 puntos estandarizados globales, lo que indica que los pacientes con insuficiencia renal crónica 
terminal manifiestan un deterioro de su calidad física, que es sensiblemente capturada por CSF. El componente de salud mental (CSM), compuesto por la agregación de las subescalas de vitalidad, función social, rol emocional y salud mental obtiene un promedio general estandarizado de 52.8 puntos que es sensiblemente superior al indicado para la salud física.

Lo anterior expresa en buenos términos lo que indica el análisis del SF-36 en su aplicación original en los datos. Los pacientes renales crónicos en tratamiento con hemodiálisis tienen problemas de salud física, que superan ampliamente las valoraciones psíquicas que hacen respecto de su propia condición de enfermos. Se podría sintetizar en que manifiestan mejor calidad de vida mental que calidad de vida física. Esta situación es muy bien expresada por los indicadores sintéticos de calidad de vida que han sido utilizados en este estudio.

Un punto sobresaliente en las conclusiones de este estudio radica en la precariedad detectada por los indicadores sintéticos, respecto de la calidad de vida física y mental del grupo de pacientes femeninos con insuficiencia renal en hemodiálisis. Si bien en términos generales los pacientes valoran más alto su calidad de vida mental que física, esto no se aplica en el caso de las pacientes mujeres. Ellas valoran peor su calidad de vida en todas las circunstancias. Particularmente las pacientes que viven en pareja tienen los peores niveles percibidos de calidad de vida física y mental, este resultado nos lleva a pensar que sería necesario profundizar en este aspecto para tener mejores antecedentes que nos permitan abordar la situación de vida de este grupo. Este hallazgo es relevante para enfermería ya que aclararlo nos permitiría proporcionar una atención más integral y adecuada al paciente y su familia.

\section{REFERENCIAS}

1. Gómez-Vela M, Sabeh E (2001). Calidad de Vida. Evolución del concepto y su influencia en la investigación y la práctica. Instituto
Universitario de Integración en la Comunidad, Facultad de Psicología, Universidad de Salamanca. Disponible en: http://campus. usal.es/ inicio/investigacion/invesinicio/ calidad.htm Acceso junio del 2006.

2. Yea-Ing, Jui Fen Rachel Lu-Jersey Leang, Evaluation of Medical Autcones Study Short Form-36 Taiwan versin assessing elderly patients with hip fracture, Osteoporos int.2004;15:575-582. Disponible :http://www.ingentaconnect.com/content/klu/198/2004/00000015/00000007/ art00010. Acceso15/nov 2007.

3. WHOQOL. Los Instrumentos de Calidad de Vida de la Organización Mundial de la Salud. Manual del Usuario, La Plata: Fundación Fundonar;1998.

4. Darja M, Sersic G, Vuletic. Psychometric Evaluation and Eslatlishing Norms of Croatian SF-36 Healtch Surwey: Framework for subjective health research. Croat Med J. Ciudad ; 2006. 95-102.

5. Schwartzmann L. Calidad de Vida. Cienc enferm. 2003; 9 (2); 1-15.

6. Olivares-Tirado P. Estado de Salud Beneficiarios del Sistema de Salud de Chile 2004-2005, Superintendencia de Isapres, Departamento de Estudios y Desarrollo 2006;. Disponible en: $<$ www.supersalud. cl/documentacion/569/articles-1062_ recurso_1.pdf $>$. Acceso el 15 abril 2007.

7. Rebollo P. Ortega F., New trends on health related quality of life assessment in endstage renal disease patients . Internacional Urology and Nephrolog; 2002, Disponible en: $<$ http://www.ingentaconnect.com/content/klu/urol/2002/00000033/00000001/00 359353 >. Acceso el 15/abril/2007

8. Guerini R, Mercieri A, Yavuzer G. Multidimensional health status assessment of.chronic hemodyalisis patients: the impact on quality of life Eura Medicophys 2006. Disponible en: <www.ncbi.nlm.nih. gov/pubmed/16767062> Acceso el 16 abril 2007

9. Yang SC, Kuo PW Kuo, Wang JD, Lin MI Su Development and Psychometric Prop- 
erties of the Diálisis Module of the Whoqol - Bref Taiwan versean J. Formos Med. 2006, Disponible en: <www.ncbi.nlm.nih. gov/pubmed/16618610> Acceso el 16 abril 2007

10.Ware J E, Gandek B. Methods for Evaluating Data Quality, Scaling Assumptions, and Reliability. J Clin Epidemiol 1998;51(11):945-952. Disponible en www. pubmedcentral.nih.gov/articlerender. fcgi?artid=1732425. Acceso el 10 marzo 2007

11.Vilagut G. Ferrer M. Raymil L. Rebollo P.Permanyer G. y cols. El Cuestionario de Salud SF-36 español: una década de experiencias y nuevos desarrollos. Gaceta Sanitaria.2006; 9(2):135-150. Disponible: $<$ ciruelo.uninorte.edu.co/pdf/salud uninorte/21/8_La\%20Calidad\%20de\%20 Vida.pdf Acceso el 15 abril 2007.

12.Ware JE, Kosinski M, Dewey J. How to Score version 2 the SF-36 ${ }^{\circledR}$ Health Survey. Lincoln, RI:Quality Metric Incorporated. 2002, Disponible en:www.lnls.br/imagem/ conteudo/425/XIV_RAU_RESUMOS.pdf. Acceso 14/nov/2007.

13.Servero M., Santos A, Lopos C, Barros H. Fiabilidad e Validade Dos Conceitos Teoricos, Das Dimensoes de Saude Fisica e
Mental Da Versao Portuguesa Do Mos sf 36. Acta Med Port. 2006;19:281-288. Disponible en: http://www.actamedicaportuguesa. com/pdf/2006-1974/281-288.pdf. Acceso 15/abril72007

14. Ware JE, Gandek B, IQOLA Project Group. The SF-36 Health Survey: Development and use inmental health research and the IQOLA Project. Int J Ment Health 1994; 23: 49-73. Disponible en: <www.scielosp.org/ scielo.php?script $=$ sci_arttext\&pid=S0213$91112005000200007 \& \operatorname{lng}>$. Acceso 10 marzo 2007.

15.Ware JE, Gandek B. Methods for Evaluating Data Quality, Scaling Assumptions, and Reliability. J Clin Epidemiology 1998;51,(11):945-952. Disponible en www. pubmedcentral.nih.gov/articlerender. fcgi?artid=1732425. Acceso el 10 marzo 2007

16.Polit y Hungler . Investigación Científica. en Ciencias de la Salud. $5^{\mathrm{a}}$ edición McGraw Hill Interamericana S.A. de C.V., Atlampa. México; 1997.

17.Dois A, Contreras A, Arechabala M, Urrutia M. Validación de una Escala de Calidad de Vida en un Grupo de Personas con Esquizofrenia de la Región Metropolitana-Chile. Cien enferm. 20007; 14(1): 35-44. 